\title{
Estimulação transcraniana por corrente contínua: da aplicação clínica ao desempenho físico
}

\author{
Transcranial direct current stimulation: \\ the clinical application to physical performance
}

Rafael A. Montenegro

Alexandre H. Okano

Sérgio Machado

Flávia Porto

Jonas L. Gurgel

Paulo T. V. Farinatti*

\section{Resumo}

Técnicas não invasivas para modular a função cerebral vêm sendo desenvolvidas, dentre as quais se destaca a estimulação transcraniana por corrente contínua (ETCC). Este artigo de revisão teve por objetivo descrever as características e mecanismos associados à ETCC, bem como suas aplicações clínicas potenciais, analisando estudos que relacionaram esta técnica com neuroplasticidade, reabilitação e desempenho físico. Os principais mecanismos de ação propostos para a ETCC envolvem receptores $\mathrm{N}$-metil-D-aspartato, principalmente no tocante aos efeitos pós-estimulação. Esses efeitos seriam influenciados por alguns neuromoduladores, como a serotonina, dopamina, adrenalina, GABA e acetilcolina. Há um consistente corpo de evidências sugerindo que a ETCC seja eficaz no tratamento de distúrbios neurológicos como doença de Parkinson, mal de Alzheimer, depressão e compulsões por drogas e alimentos. Estudos recentes sugerem que um novo

Revista HUPE, Rio de Janeiro, 2013;12(4):27-37 doi:110.12957/rhupe.2013.8710 campo de aplicabilidade da ETCC possa estar relacionado com um provável efeito ergogênico, especialmente sobre a produção de força muscular, desempenho aeróbio e percepção do esforço. É possível, igualmente, que a ETCC possa ter efeitos favoráveis sobre o dispêndio energético após exercício físico e com o controle da pressão arterial.

Descritores: Terapia por estimulação elétrica; Neurotransmissores; Efeito melhoria do rendimento; Exercício físico; Saúde.

\begin{abstract}
Non-invasive techniques to modulate brain function have been developed, among which the transcranial direct current stimulation (tDCS). The present review aimed to describe the characteristics and mechanisms related to tDCS, as well as its potential clinical applications, analyzing research that investigated the relationship of this technique with neuroplasticity, physical rehabilitation and
\end{abstract}

"Endereço para correspondência: Laboratório de Atividade Física e Promoção da Saúde, UERJ Rua São Francisco Xavier 524, 80 andar, sala 8133, bloco F Rio de Janeiro, RJ, Brasil. CEP: 20599-900 E-mail: paulo.farinatti@pq.cnpq.br 
physical performance. The main mechanisms for the tDCS modulation have been related to $\mathrm{N}$-methyl-D-aspartate receptors, especially with regard to the post-stimulation effects. Such effects would also be influenced by neuromodulators such as serotonin, dopamine, adrenaline, GABA and acetylcholine. Accumulated evidence suggests that tDCS can be effectively applied in the treatment of neurological disturbs as the Parkinson's Disease, Alzheimer, depression, and cravings for drugs and food. More recent studies have examined its potential applicability as an ergogenic strategy, increasing the muscle strength during specific tasks, aerobic capacity, and decreasing the rate of perceived exertion. It has been also proposed that the tDCS may influence favorably the energy expenditure and blood pressure after physical exercise.

Keywords: Electric stimulation therapy; Neurotransmitter agents; Performance-enhancing substances; Exercise; Health.

\section{Introdução}

Técnicas não invasivas para modular a função cerebral vêm sendo desenvolvidas, dentre as quais se destaca a estimulação transcraniana por corrente contínua (ETCC). ${ }^{1}$

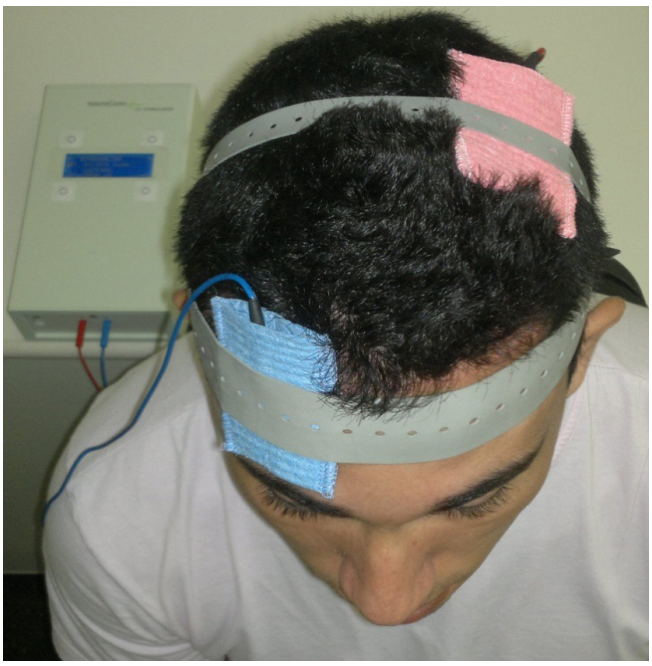

Figura 1. Montagem bicefálica da ETCC

O eletrodo envolvido com a esponja de cor vermeIha encontra-se sobre o córtex motor esquerdo, já o eletrodo com a esponja de cor azul está sobre o córtex supraorbital.
Esta técnica se compõe por equipamentos de baixo custo e simples manuseio. Eletrodos de silicone envolvidos por esponja embebida em solução salina são posicionados sobre o córtex que se deseja estimular. Uma corrente elétrica contínua que varia entre 0,4-2 miliampères é imposta sobre a região cortical por um período de 3-20 minutos (Figura 1). Por meio deste procedimento modificações na excitabilidade cortical têm sido alcançadas. ${ }^{2}$ A depender da polaridade da corrente elétrica escolhida, efeitos diferentes são desencadeados. Por exemplo, com o estímulo de corrente anódica um aumento na excitabilidade cortical tem sido relatado, enquanto que o estímulo de corrente catódica promove efeito oposto (Figura 2). ${ }^{2}$

Nas últimas décadas, a ETCC vem sendo utilizada tanto no campo clínico e terapêutico quanto no científico experimental. Portanto, um vasto campo de aplicabilidade tem sido documentado, estendendo-se ao tratamento de diferentes distúrbios neurológicos. ${ }^{3}$ No entanto, relatos apontam não apenas para a efetividade clínica e terapêutica da técnica, mas também para a possibilidade de acarretar melhorias no desempenho físico e metabolismo, gerenciamento da pressão arterial e controle autonômico cardíaco, ${ }^{4,5}$ podendo ser futuramente utilizado como um importante recurso ergogênico para modalidades esportivas. ${ }^{6}$

Assim, estudos vêm sendo realizados com o intuito de melhorar a compreensão acerca dos possíveis mecanismos envolvidos nas modificações induzidas pela ETCC. Contudo, apesar do crescente número de estudos que visam explicar os mecanismos de ação envolvidos nos efeitos em longo prazo após a ETCC, ainda não está bem documentada na literatura a relação entre os canais iônicos, receptores de membrana e os efeitos observados após a ETCC, bem como a relação entre a neuromodulação e aumentos na força muscular e desempenho aeróbio.

Diante do exposto, este artigo de revisão tem como objetivo relatar o histórico da estimulação cerebral e mecanismo de ação, bem 


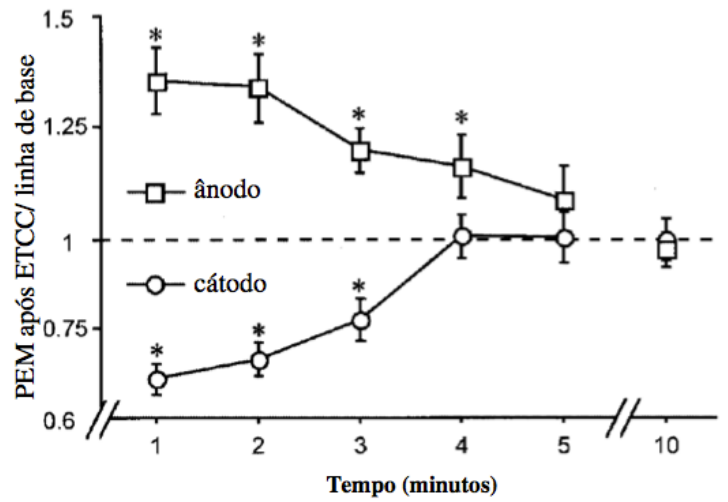

Figura 2. Efeitos pós-estimulatórios da ETCC anódica e catódica aplicada por 5 minutos a $1 \mathrm{~mA}$ Os efeitos pós-estimulatórios duraram cerca de 5 minutos.

Fonte: Adaptado de Excitability changes induced in the human motor cortex by weak transcranial direct current stimulation. ${ }^{2}$

como as aplicações clínicas da ETCC e analisar os estudos que relacionam esta técnica com neuroplasticidade, reabilitação e desempenho físico.

\section{Estimulação transcraniana}

\section{Principais técnicas de neuromodulação}

A utilização de corrente elétrica para fins terapêuticos é antiga, remontando ao século I. Descargas elétricas de peixes elétricos eram aplicadas na cabeça de pacientes, desencadeando efeitos entorpecentes e aliviando dores de cabeça. ${ }^{7}$ No decorrer dos tempos e sob diversas formas, a estimulação cerebral por meio de correntes elétricas consistiu em estratégia terapêutica bastante utilizada para minimizar os efeitos de distúrbios mentais.

Mais recentemente, os avanços tecnológicos na área da neurologia suscitaram um interesse crescente em técnicas não invasivas para modulação da polarização cerebral. Atualmente, duas técnicas de neuromodulação têm posição de destaque: a estimulação magnética transcraniana (EMT) e a estimulação transcraniana por corrente contínua (ETCC). A EMT foi patenteada por Pollacsek e Beer em 1902, com o propósito da aplicação de campos eletromagnéticos sobre o couro cabeludo, para o tratamento da depressão e neurose. Também era usada para estudos de mapeamento do córtex motor, podendo ser aplicada, de forma totalmente não invasiva e indolor, no ser humano desperto. O primeiro aparelho moderno de EMT foi desenvolvido por Anthony Barker em $1985 .{ }^{8}$ Esta técnica é regulada por meio do ajuste adequado da frequência e intensidade dos impulsos magnéticos. O impulso magnético é gerado por uma bobina posicionada sobre a região cortical que será estimulada ou inibida. A utilização de altas frequências é capaz de induzir um aumento duradouro da excitabilidade neuronal da região em foco, ao passo que a EMT de baixa frequência, inversamente, induz uma redução de excitabilidade cortical que perdura por horas ou dias após a aplicação dos estímulos. Porém, a EMT necessita de um mecanismo de refrigeração após o aquecimento da bobina quando utilizada em altas frequências, gerando assim um alto custo para seu manuseio.

A ETCC consiste em técnica de neuromodulação mais simples e menos dispendiosa, sendo capaz de induzir mudanças na excitabilidade do córtex motor por até uma hora após o final da estimulação. ${ }^{2}$ Tais mudanças dependem do tipo de polaridade da corrente elétrica que está sendo aplicada e do tempo de duração do estímulo. A polaridade de corrente anódica é responsável por aumentar a excitabilidade cortical, ao passo que a polaridade catódica é responsável por efeitos contrários, 
suprimindo a excitabilidade cortical. ${ }^{2}$

\section{Mecanismos de ação da ETCC}

Em um dos primeiros estudos com a técnica, Nitsche e Paulus ${ }^{2}$ verificaram os efeitos e duração da ETCC anódica e catódica aplicada sobre o córtex motor. Para tanto, utilizou-se a medida do potencial motor evocado (PEM) em dois diferentes protocolos de estimulação, quais sejam 1 e 5 minutos com intensidade de 0,2 e 1,0 mA, respectivamente. Observou-se que ETCC anódica com duração de 5 min aumentou a excitabilidade cortical, incrementando a amplitude do PEM em torno de $40 \%$ após a estimulação, enquanto a catódica provocou uma redução de 30\% do PEM.

Vale ressaltar que a duração desses efeitos dependeu do tempo de aplicação e intensidade da corrente, ou seja, a ETCC aplicada por 5 minutos gerou modificações na excitabilidade neuronal que permaneceram por esse mesmo tempo após o término da ETCC anódica, porém para a estimulação catódica o mesmo não ocorreu. Nitsche e Paulus ${ }^{2}$ explicaram esses resultados com base na despolarização neural causada pela estimulação anódica, incrementando a excitabilidade neural. Por outro lado, a estimulação catódica causaria uma hiperpolarização, com efeito inibitório sobre os neurônios. No entanto, no âmbito celular poucos estudos almejaram buscar explanações a respeito da relação entre canais iônicos, receptores e efeitos observados durante e após diferentes condições de estimulação cortical.

Liebetanz e colaboradores ${ }^{9}$ estudaram os efeitos da ETCC aplicada sobre o córtex motor em condição anódica ou catódica, observando possíveis interferências de bloqueador de canais de sódio dependentes de voltagem (carbamazepina - CBZ) e antagonista de receptor $\mathrm{N}$-metil-D-aspartato (NMDA) (dextrometorfana - DMO) sobre os efeitos da estimulação. Assim como Nitsche e Paulus, ${ }^{2}$ observou-se que a ETCC anódica resultou em aumento na excitabilidade cortical, enquanto a ETCC catódica a diminuiu. Porém, a administração de DMO suprimiu os efeitos pós-estimulação, tanto catódica quanto anódica. Assim, sugeriu-se o envolvimento dos receptores NMDA nos mecanismos de plasticidade neuronal induzidos pelos dois tipos da ETCC. Já a CBZ suprimiu apenas os efeitos da ETCC anódica, indicando uma maior participação dos canais de sódio nesse tipo de estimulação.

No ano seguinte, Nitsche e colaboradores ${ }^{10}$ deram continuidade a sua hipótese, investigando os efeitos da ETCC ao administrar CBZ e DMO, acrescentando um novo grupo de indivíduos que receberam flunarizina (FLU), bloqueador de canais de cálcio. Assim, verificouse a importância de outros canais iônicos nos mecanismos de ação da ETCC. A observação em relação aos canais de cálcio se deu em função da sua importância nos processos de liberação de neurotransmissores. Foram analisados os efeitos em curto prazo (ETCC de 4 segundos) e em médio prazo (entre 9 e 13 minutos após a aplicação da ETCC). No tocante aos efeitos em curto prazo, o bloqueio de canais de sódio eliminou completamente o aumento na excitabilidade observada durante a estimulação anódica e o bloqueio dos canais de cálcio apenas diminuiu esse aumento. Entretanto, o bloqueio desses canais iônicos não produziu interferência nos efeitos normais de diminuição da excitabilidade cortical após estimulação catódica. Os autores consideraram que, em função do efeito supressor da estimulação catódica ser atribuído a uma hiperpolarização da membrana neuronal, a administração de bloqueadores dos canais de sódio e cálcio não resultaria, de fato, em nenhum efeito.

Além disso, o antagonista do receptor NMDA interferiu tanto no aumento da excitabilidade cortical (estimulação anódica), quanto na redução promovida pela ETCC catódica. Nitsche e colaboradores ${ }^{10}$ sugeriram que isso refletiria a importância desses receptores na promoção dos efeitos em longo prazo, principalmente quando se considera o crescente conhecimento sobre o envolvimento dos 
receptores NMDA em relação aos mecanismos de plasticidade cortical, como os de potenciação de longo prazo (PLP) ou depressão de longo prazo (DLP).

Também existem indícios do possível envolvimento de outros componentes do sistema glutamatérgico nos efeitos da ETCC. Considerando que tais efeitos são originados intracorticalmente, o ácido gama-aminoburítico (GABA) seria um forte candidato para desempenhar este papel, pois os neurônios GABAérgicos são inibitórios e predominam no córtex cerebral. ${ }^{11}$ Para avaliar essa influência, Nitsche e colaboradores ${ }^{11}$ hipotetizaram que a aplicação de um agonista do GABA (Lorazepam - LOR) poderia comprometer a ação dos receptores NMDA e diminuir o efeito em longo prazo observado após a ETCC anódica. Por outro lado, não se esperaria nenhuma influência sobre os efeitos já documentados da estimulação catódica. Os resultados revelaram que a ETCC catódica aplicada em conjunto com a droga não diferiu da condição placebo. Já a estimulação anódica apresentou um retardo inicial na amplitude do PEM, seguido de um aumento da amplitude e prolongação dos efeitos até os primeiros 60 minutos pós-estimulação, indicando uma participação do GABA na neuroplasticidade cortical.

Buscando consolidar o papel do receptor NMDA na neuroplasticidade, Nitsche e colaboradores $^{12}$ verificaram os efeitos da administração de $D$-cycloserine, agonista parcial deste receptor. A ação da droga associada com a ETCC anódica aumentou a excitabilidade neuronal até a manhã do dia seguinte à estimulação, porém não houve efeito sobre a amplitude do PEM. Resultados semelhantes foram encontrados pelo mesmo grupo de autores ${ }^{13}$ em resposta à anfetamina. Com administração do propanolol, antagonista $\beta$-adrenérgico, ocorreu diminuição dos efeitos pós-ETCC anódica e catódica, indicando que uma determinada quantidade de atividade adrenérgica parece ser necessária para consolidação de ambos os mecanismos de neuroplasticidade.

\section{ETCC aplicada ao tratamento de doenças neurológicas e transtornos psiquiátricos}

Processos de plasticidade cerebral duradouros dependem de mudanças que ocorrem diretamente na força sináptica, como alterações nas espinhas dendríticas. Uma vez que essas alterações anatômicas resultam, secundariamente, em alterações prolongadas na força sináptica, a função da ETCC é alterar a força sináptica. ${ }^{14} \mathrm{Tal}$ efeito tem sido observado tanto em doenças neurológicas quanto psiquiátricas, promovendo melhorias às áreas motoras e comportamentais. Dessa forma, nesta seção, apresentam-se os principais achados relacionados com os potenciais efeitos terapêuticos da ETCC em doenças neurológicas e psiquiátricas.

\section{ETCC, doença de Parkinson e Alzheimer}

A doença de Parkinson (DP) é caracterizada pelo envolvimento de múltiplas vias neuroanatômicas com algumas das características clínicas resultantes da degeneração dos neurônios dopaminérgicos na substância negra. A degeneração das vias dopaminérgicas resulta em falhas nas aferências de alvos funcionais do córtex cerebral que provavelmente contribuem para a fisiopatologia de distúrbios motores e não motores em pacientes com DP.

Os fatores investigados pela neuromodulação com ETCC na DP incluem aqueles que induzem a liberação de dopamina e que podem normalizar a excitabilidade cortical anormal ou atividade de redes neurais. Até o momento, foram realizados apenas dois estudos sobre ETCC e DP. No primeiro estudo, Fregni e colaboradores ${ }^{15}$ analisaram os efeitos de uma única sessão de ETCC, aplicada no córtex préfrontal dorsolateral (CPFDL) esquerdo e no córtex motor primário (M1) em pacientes com 
DP que não faziam uso de medicação. A ETCC anódica aplicada em M1 resultou em melhoria significativa da função motora na $\mathrm{DP}$, quando comparada com a ETCC placebo.

No segundo estudo, demonstrou-se uma melhoria significativa na memória de trabalho de pacientes com DP, ${ }^{16}$ após a aplicação de ETCC anódica no CPFDL esquerdo com $2 \mathrm{~mA}$. As outras condições de estimulação (quais sejam ETCC placebo, ETCC anódica aplicada no CPFDL esquerdo com $1 \mathrm{~mA}$ ou ETCC anódica aplicada em M1) não resultaram em mudança significativa no desempenho da tarefa. Esses resultados sugerem não somente especificidade do local de aplicação da ETCC, mas também especificidade da dose de corrente aplicada (1 $\mathrm{mA}$ vs. $2 \mathrm{~mA}$ ).

A doença de Alzheimer (DA) é uma das causas mais comuns de demência, sendo caracterizada por deficit secundário de memória pela degeneração no sistema límbico.

Foram realizados apenas dois estudos com ETCC para melhorar a memória de reconhecimento em pacientes com DA. ${ }^{17,18}$ Devido ao padrão hipoativado das áreas temporoparietais (CTP) em pacientes com DA, Ferrucci e colaboradores ${ }^{18}$ conduziram um estudo-piloto para verificar se ETCC anódica aplicada sobre esta área poderia melhorar a memória de reconhecimento em 10 pacientes com DA leve. Foram aplicadas ETCC anódica, catódica, e placebo sobre o CTP em três sessões diferentes (15 min a 1,5 mA), com pelo menos uma semana de intervalo entre cada aplicação. A ETCC anódica melhorou significativamente a memória de reconhecimento, enquanto a ETCC catódica diminuiu significativamente a precisão na tarefa de reconhecimento de palavras.

No segundo estudo, Boggio e colaboradores ${ }^{17}$ investigaram os efeitos da ETCC anódica aplicada sobre o córtex temporal (CT) e CPFDL esquerdo (30 min a $2 \mathrm{~mA}$ ) na memória de reconhecimento, memória de trabalho e atenção seletiva em pacientes com DA leve a moderadamente severa. A ETCC anódica resultou na melhoria significativa da memória de reconhecimento visual, mas nenhum efeito foi observado na memória de trabalho.

Tais resultados fazem com que a ETCC se torne particularmente relevante, já que permite a facilitação da plasticidade neuronal por indução de efeitos de longa duração. Os poucos estudos realizados até o presente momento revelam efeitos positivos e fornecem evidências iniciais sobre o potencial da ETCC para a melhoria de determinadas funções cognitivas e retardo da progressão da DA. No entanto, esses estudos não foram replicados e as evidências ainda são preliminares.

\section{ETCC, depressão e compulsão por drogas e alimentos}

A depressão maior (DM) é um transtorno de humor caracterizado por disfunções afetivas, comportamentais e cognitivas. Estudos de neuroimagem geralmente demonstram atividade reduzida no córtex pré-frontal $(\mathrm{CPF})$ de pacientes com DM, especialmente no CPF esquerdo (áreas 9 e 46 de Broadmann), e ativação anormal no circuito corticossubcortical compreendido pelos córtices cingulado anterior e subgenual.

Com relação ao tratamento da DM com ETCC, apenas cinco estudos foram localizados. O primeiro foi realizado por Fregni e colaboradores, ${ }^{19}$ os quais administraram um protocolo de cinco sessões de ETCC anódica de $1 \mathrm{~mA}$ sobre o CPFDL esquerdo, constatando melhora na memória de trabalho. Boggio e colaboradores $^{3}$ administraram dez sessões de ETCC anódica a $2 \mathrm{~mA}$, também sobre o CPFDL esquerdo. Observou-se melhora de desempenho, com aumento de respostas corretas para figuras com conteúdo emocional positivo. Já Loo e colaboradores ${ }^{20}$ não relataram mudança no desempenho cognitivo decorrente da ETCC em um estudo, mas observaram efeito agudo positivo na atenção e memória de trabalho em outro, ${ }^{21}$ mas apenas agudamente. Nenhum efeito crônico ocorreu após 15 sessões de ETCC anódica $(2 \mathrm{~mA})$ em 64 pacientes, apesar de ser observado depois de sessões isoladas. 
Já as compulsões ou cravings por álcool, drogas lícitas ou ilícitas e alimentos têm sido objeto de pesquisa de vários estudos científicos recentes. Os sinais que induzem o desejo descontrolado ou compulsão são afetados por uma variedade de contextos e eventos que surtem impacto sobre o autocontrole, tais como a percepção da disponibilidade de drogas ou outra substância, a expectativa, o estresse, ou a intenção de procurar tratamento. Um grande corpo de evidências aponta para que a origem das compulsões seja a partir da associação de redes neurais com o processo de tomada de decisão. Nesse sentido, com a manipulação da atividade neuronal do CPFDL por meio da TMS e ETCC, foi possível reduzir o desejo pelo cigarro, cocaína, álcool e alimentos. ${ }^{22,23}$

A atividade física tem estreita relação com a ingestão alimentar, pois o trabalho corporal requer a energia fornecida pelos nutrientes para que haja restauração dos estoques de combustível, com o propósito da continuidade do funcionamento das funções corporais. Mayer e colaboradores ${ }^{24}$ defendem a hipótese de que, imediatamente após a um significativo aumento no dispêndio energético, um balanço energético negativo é gerado. De acordo com esse modelo, vários autores corroboram a ideia de que haveria um efeito supressor da ingestão alimentar como resposta reativa às séries agudas de atividades físicas.

Com base nisso, recentemente nosso grupo demonstrou que a ETCC aplicada sobre o CPFDL suprime o desejo pelo alimento. Quando associados aos efeitos de uma sessão de exercício aeróbio de intensidade moderada, a ETCC anódica aumentou ainda mais o efeito anoréxico comparado aos efeitos isolados da ETCC anódica ou exercício aeróbio. Além disso, esses efeitos anoréxicos perduraram por até 30 minutos após o término do exercício físico. ${ }^{25}$ Estudos futuros devem investigar o quanto esse tipo de efeito pode ajudar no controle da ingestão alimentar em programas de controle de peso que combinem dieta e exercício físico.

\section{Efeito da ETCC na reabilitação e desempenho físico}

A plasticidade neural é uma propriedade fisiológica do cérebro que permite que se adapte a diferentes estímulos. Está, por isso, diretamente ligada aos processos de aprendizagem e desenvolvimento de tarefas. É essa capacidade do sistema nervoso central (SNC) que permite, por exemplo, que amputados consigam, com treinamento, dar ao membro que permaneceu no corpo a mesma desenvoltura daquele que foi perdido, que possibilita a reabilitação motora, cognitiva e de fala de pessoas que tiveram acidente vascular encefálico (AVE) ou, ainda, que explica o declínio funcional provocado pelo processo de envelhecimento. Os efeitos neuroplásticos da ETCC também têm sido mostrados na área de desempenho físico. Nesse caso, melhorias foram observadas em relação ao aumento da produção de força e na capacidade de manter determinada carga durante um período, bem como na melhora da capacidade aeróbia. ${ }^{5}$

A importância dos componentes da aptidão cardiorrespiratória e musculoesquelética (ou seja, a capacidade de gerar força máxima muscular, resistência de força muscular e flexibilidade) é evidente, pois estão relacionados com a saúde funcional e o bem-estar das pessoas. O objetivo do emprego da ETCC, neste campo, é promover efeitos modulatórios da fadiga por meio da modulação do potencial de repouso da membrana neuronal, incrementando-se a possibilidade de se induzir efeitos benéficos com o treinamento físico. Além disso, a ETCC pode influenciar os mecanismos autonômicos do SNC, refletindo-se no controle do sistema cardiovascular, inclusive durante exercícios físicos. ${ }^{4,5}$

Se os efeitos da ETCC também dependem do local de colocação dos eletrodos, nesta área de atuação o estímulo tem sido aplicado na região do córtex motor e do córtex insular. No primeiro caso, vários estudos têm mostrado benefícios do uso da ETCC, sobretudo da corrente anódica, 
quando aplicada na área do córtex motor (M1). Coggiamanian e colaboradores ${ }^{26}$ verificaram que a corrente anódica propiciou aumento da força isométrica de músculos flexores de cotovelo a $35 \%$ da contração voluntária máxima. Já Tanaka e colaboradores ${ }^{27}$ mostraram que a mesma corrente provocou aumento da produção de torque no movimento de pinçar, tanto dos dedos das mãos quanto dos pés. Isso demonstra que a ETCC parece influenciar tanto as regiões mais superficiais do cérebro - responsáveis pelo movimento dos membros superiores - como as regiões mais profundas, que inervam os músculos dos membros inferiores.

A ETCC tem se mostrado uma técnica promissora de auxílio à reabilitação motora. Madhavan e colaboradores, ${ }^{28}$ por exemplo, verificaram que pacientes neurológicos melhoraram mais rapidamente a execução de movimentos de flexão e extensão de tornozelo, após sua aplicação. No caso de pacientes lesionados no cérebro, o conceito de competição inter-hemisférica é considerado para o entendimento e emprego da ETCC, já que nesses indivíduos há um mau funcionamento dos hemisférios corticais e um desajuste na inibição inter-hemisférica. Isso explica os efeitos positivos encontrados no estudo de Madhavan e colaboradores, ${ }^{28}$ no qual uma corrente anódica aplicada sobre o hemisfério lesionado e uma corrente catódica aplicada no hemisfério sadio promoveram aumento das funções motoras de pacientes com acidente vascular encefálico.

No que tange às relações com a aptidão cardiorrespiratória, o controle autonômico cardiovascular também parece ser modulado pela ETCC. Estudo realizado por nosso grupo verificou que a corrente anódica aplicada sobre o lobo temporal (T3) (almejando, portanto, estimular o córtex insular) promoveu aumento da variabilidade da frequência cardíaca de atletas, sugerindo uma melhoria do controle autonômico dos indivíduos. ${ }^{4}$ Mais recentemente, nosso grupo também demonstrou o efeito ergogênico gerado pela ETCC anódica aplicada no T3 sobre o limiar de variabilidade da frequência cardíaca, carga máxima atingida e tempo de exaustão de ciclistas bem-treinados. ${ }^{5}$ Todavia, em dados ainda não publicados, nosso grupo demonstrou que a ETCC anódica aplicada sobre o córtex motor parece não aumentar os níveis máximos de força muscular, medidos em exercício unilateral de extensão e flexão de joelhos em indivíduos adultos saudáveis. Por outro lado, apesar da produção de força muscular ter sido a mesma, as respostas pressóricas foram atenuadas em comparação com a ETCC placebo. Com base nesses achados, é factível pensar que a aplicação de ETCC em grupos de hipertensos pode diminuir os picos pressóricos gerados pelo exercício de força, sem que haja prejuízos na geração de força muscular. Tais estudos parecem ser os pioneiros na área e, na verdade, estudos sobre efeitos da ETCC e desempenho físico, principalmente de atletas, ainda são escassos, o que dificulta o entendimento dos mecanismos envolvidos da ETCC sobre a fadiga e o desempenho físico. Certamente, trata-se de uma área que merece maior aprofundamento em investigações futuras.

No tocante às respostas após exercício físico, o gasto energético após exercício aeróbio, também denominado de consumo de oxigênio excessivo pós-exercício (EPOC), tem sido bastante estudado. ${ }^{29}$ Exercícios físicos que promovam um maior EPOC são interessantes para indivíduos que almejam um maior balanço energético negativo, o qual repercutirá na redução do peso corporal. ${ }^{30}$ Nesse contexto, nosso grupo publicou recentemente um estudo pioneiro na investigação da influência da modulação neuronal do CPFDL e seus efeitos sobre o EPOC após sessões isocalóricas de exercício aeróbio. Depois da aplicação de ETCC anódica sobre o CPFDL, o EPOC foi aumentado em aproximadamente $19 \%$ comparado à sessãocontrole. Com isso, os autores concluíram que as modificações neuronais sobre o CPFDL - córtex responsável pelo controle autônomo respiratório, desencadeadas por meio da ETCC anódica combinada com exercício aeróbio 
submáximo - foram capazes de aumentar o gasto calórico e o consumo de oxigênio durante o exercício e por, pelo menos, 30 minutos após o término da sessão de treino. ${ }^{29}$ Em suma, acredita-se que a melhoria do desempenho, principalmente aeróbio, estaria relacionada à diminuição da percepção subjetiva de esforço devido à estimulação cortical e por efeito indireto sobre áreas modulatórias de dor.

Levando em consideração que todas as evidências expostas baseiam-se no efeito agudo gerado pela ETCC, estudos sobre o efeito crônico da aplicação de ETCC nos marcadores de desempenho físico se fazem necessários. Até onde foi nosso esforço de revisão, localizou-se apenas um estudo recémpublicado investigando o efeito de múltiplas sessões (9) de ETCC sobre o desempenho de força dos músculos extensores da articulação do punho. ${ }^{31}$ Nesse estudo, a amostra foi dividida em três grupos iguais de 10 indivíduos, dos quais um grupo recebeu a ETCC anódica, outro recebeu a ETCC placebo e o terceiro serviu de controle. Além disso, os grupos experimentais realizaram treinos resistidos (3 séries de 6-8 repetições a 70\% de 1 repetição máxima). Após as nove sessões do protocolo experimental, os autores concluíram que os níveis máximos de força muscular foram aumentados em ambos os grupos experimentais em comparação ao grupo-controle. Porém nenhuma diferença estatística foi apontada quando foi comparado o grupo da ETCC anódica com o da ETCC placebo. Todavia, somente o grupo que recebeu ETCC anódica aumentou a excitabilidade corticoespinal. Apesar de o estudo de Hendy e Kidgell ${ }^{31}$ não ter demonstrado efeitos benéficos sobre os níveis máximos de força muscular, outros estudos crônicos investigando os efeitos da ETCC sobre níveis de força de outros grupamentos musculares maiores, bem como sobre o condicionamento cardiorrespiratório devem ser incentivados.

\section{Considerações finais}

Dentre as técnicas não invasivas para modular a função cerebral, destaca-se a ETCC. Seus principais mecanismos de ação parecem estar relacionados com receptores N-metil-Daspartato (NMDA), principalmente no tocante aos efeitos pós-estimulação. Esses efeitos seriam influenciados por alguns neuromoduladores, como a serotonina, dopamina, adrenalina, GABA e acetilcolina. Um crescente corpo de evidências vem sendo formado relatando a eficácia clínica da ETCC no tratamento de distúrbios neurológicos como Alzheimer, Parkinson, depressão crônica e desejos abusivos por substâncias como drogas, álcool e alimento.

Estudos recentes sugerem que um novo campo de aplicabilidade da ETCC possa estar relacionado com um provável efeito ergogênico por ela induzido, especialmente sobre a produção de força muscular, desempenho aeróbio e percepção do esforço. É possível, igualmente, que a ETCC possa ter efeitos favoráveis sobre o dispêndio energético após exercício físico e com o controle da pressão arterial. Estudos adicionais devem ser estimulados para confirmação do potencial clínico da técnica em diferentes dimensões, bem como para a elucidação de mecanismos envolvidos em tais efeitos.

\section{Referências}

1. Nitsche MA, Paulus W. Transcranial direct current stimulation - update 2011. Restor Neurol Neurosci. 2011;29(6):46392. http://dx.doi.org/10.3233/RNN-20110618252R1135VU705H53

2. Nitsche MA, Paulus W. Excitability changes induced in the human motor cortex by weak transcranial direct current stimulation. J Physiol. 2000 Sep 15;527(3):633-9 http:// dx.doi.org/PHY_1055

3. Boggio PS, Bermpohl F, Vergara AO, Muniz AL, Nahas FH, Leme PB, et al. Go-nogo task performance improvement after anodal transcranial DC stimulation of the left dorsolateral prefrontal cortex in major depression. J Affect Disord. 2007 Aug;101(13):91-8. http://dx.doi.org/PHY_1055S01650327(06)00466-610.1016/j.jad.2006.10.026

4. Montenegro RA, Farinatti PT, Fontes EB, Soares PP, Cunha FA, Gurgel JL, et al. Transcranial direct current stimulation influences the cardiac autonomic nervous control. Neurosci Lett. 2011 Jun 15;497(1):32- 
6. http://dx.doi.org/PHY_1055S0304-

3940(11)00465-410.1016/j.neulet.2011.04.019

5. Okano AH, Fontes EB, Montenegro RA, Farinatti PD, Cyrino ES, Li LM, et al. Brain stimulation modulates the autonomic nervous system, rating of perceived exertion and performance during maximal exercise. Br J Sports Med. 2013 Feb 27. http:// dx.doi.org/bjsports-2012-09165810.1136/ bjsports-2012-091658

6. Vitor-Costa M, Pereira LA, Montenegro RA, Okano AH, Altimari LR. A estimulação transcraniana por corrente contínua como recurso ergogênico: uma nova perspectiva no meio esportivo. Rev Educ Fis/UEM. 2012;23(2):167-74.

7. Priori A. Brain polarization in humans: a reappraisal of an old tool for prolonged noninvasive modulation of brain excitability. Clin Neurophysiol. 2003 Apr;114(4):589-95.

8. Barker AT, Jalinous R, Freeston IL. Non-invasive magnetic stimulation of human motor cortex. Lancet. 1985 May 11;1(8437):1106-7. http://dx.doi.org/S01406736(85)92413-4

9. Liebetanz D, Nitsche MA, Tergau F, Paulus W. Pharmacological approach to the mechanisms of transcranial DC-stimulationinduced after-effects of human motor cortex excitability. Brain. 2002 Oct;125(10):2238-47.

10. Nitsche MA, Fricke K, Henschke U, Schlitterlau A, Liebetanz D, Lang N, et al. Pharmacological modulation of cortical excitability shifts induced by transcranial direct current stimulation in humans. J Physiol. 2003 Nov 15;553(1):293-301. http:// dx.doi.org/10.1113/jphysiol.2003.049916jphysi ol.2003.049916.

11. Nitsche MA, Liebetanz D, Schlitterlau A, Henschke U, Fricke K, Frommann $\mathrm{K}$, et al. GABAergic modulation of DC stimulation-induced motor cortex excitability shifts in humans. Eur J Neurosci. 2004 May;19(10):2720-6. http://dx.doi.org/10.1111/ j.0953-816X.2004.03398.xEJN3398

12. Nitsche MA, Jaussi W, Liebetanz D, Lang N, Tergau F, Paulus W. Consolidation of human motor cortical neuroplasticity by D-cycloserine. Neuropsychopharmacology. 2004 Aug;29(8):1573-8. http://dx.doi. org/10.1038/sj.npp.13005171300517

13. Nitsche MA, Grundey J, Liebetanz D, Lang N, Tergau F, Paulus W. Catecholaminergic consolidation of motor cortical neuroplasticity in humans. Cereb Cortex. 2004 Nov;14(11):1240-5. http://dx.doi. org/10.1093/cercor/bhh085bhh085
14. Nitsche MA, Cohen LG, Wassermann EM, Priori A, Lang N, Antal A, et al. Transcranial direct current stimulation: State of the art 2008. Brain Stimul. 2008 Jul;1(3):206-23. http:// dx.doi.org/S1935-861X(08)00040-510.1016/j. brs.2008.06.004

15. Fregni F, Boggio PS, Santos MC, Lima M, Vieira AL, Rigonatti SP, et al. Noninvasive cortical stimulation with transcranial direct current stimulation in Parkinson's disease. Mov Disord. 2006 Oct;21(10):1693-702. http:// dx.doi.org/10.1002/mds.21012

16. Boggio PS, Ferrucci R, Rigonatti SP, Covre P, Nitsche M, Pascual-Leone A, et al. Effects of transcranial direct current stimulation on working memory in patients with Parkinson's disease. J Neurol Sci. 2006 Nov 1;249(1):318. http://dx.doi.org/S0022-510X(06)00280210.1016/j.jns.2006.05.062

17. Boggio PS, Khoury LP, Martins DC, Martins OE, de Macedo EC, Fregni F. Temporal cortex direct current stimulation enhances performance on a visual recognition memory task in Alzheimer disease. J Neurol Neurosurg Psychiatry. 2009 Apr;80(4):444-7. http://dx.doi.org/10.1136/jnnp.2007.141853

18. Ferrucci R, Mameli F, Guidi I, Mrakic-Sposta S, Vergari M, Marceglia S, et al. Transcranial direct current stimulation improves recognition memory in Alzheimer disease. Neurology. 2008 Aug 12;71(7):493-8. http:// dx.doi.org/10.1212/01.wnl.0000317060.43722.a3

19. Fregni F, Boggio PS, Nitsche MA, Rigonatti SP, Pascual-Leone A. Cognitive effects of repeated sessions of transcranial direct current stimulation in patients with depression. Depress Anxiety. 2006;23(8):4824. http://dx.doi.org/10.1002/da.20201

20. Loo CK, Sachdev P, Martin D, Pigot M, Alonzo A, Malhi GS, et al. A double-blind, sham-controlled trial of transcranial direct current stimulation for the treatment of depression. Int J Neuropsychopharmacol. 2010 Feb;13(1):61-9. http://dx.doi.org/10.1017/ S1461145709990411

21. Loo CK, Alonzo A, Martin D, Mitchell PB, Galvez V, Sachdev P. Transcranial direct current stimulation for depression: 3-week, randomised, sham-controlled trial. Br J Psychiatry. 2012 Jan;200(1):52-9. http://dx.doi. org/200/1/5210.1192/bjp.bp.111.097634

22. Boggio PS, Sultani N, Fecteau S, Merabet L, Mecca T, Pascual-Leone A, et al. Prefrontal cortex modulation using transcranial DC stimulation reduces alcohol craving: a double-blind, sham-controlled study. Drug Alcohol Depend. 2008 Jan 1;92(1-3):55-60. http://dx.doi.org/S0376-8716(07)00250510.1016/j.drugalcdep.2007.06.011 
23. Eichhammer P, Johann M, Kharraz A, Binder H, Pittrow D, Wodarz N, et al. Highfrequency repetitive transcranial magnetic stimulation decreases cigarette smoking. J Clin Psychiatry. 2003 Aug;64(8):951-3.

24. Mayer J, Roy P, Mitra KP. Relation between caloric intake, body weight, and physical work: studies in an industrial male population in West Bengal. Am J Clin Nutr. 1956 Mar-Apr;4(2):169-75.

25. Montenegro RA, Okano AH, Cunha FA, Gurgel JL, Fontes EB, Farinatti PT. Prefrontal cortex transcranial direct current stimulation associated with aerobic exercise change aspects of appetite sensation in overweight adults. Appetite. 2012 Feb;58(1):333-8. http:// dx.doi.org/S0195-6663(11)00637-410.1016/j. appet.2011.11.008

26. Cogiamanian F, Marceglia S, Ardolino G, Barbieri S, Priori A. Improved isometric force endurance after transcranial direct current stimulation over the human motor cortical areas. Eur J Neurosci. 2007 Jul;26(1):242-9. http://dx.doi.org/EJN563310.1111/j.14609568.2007.05633.x

27. Tanaka S, Hanakawa T, Honda M, Watanabe
K. Enhancement of pinch force in the lower leg by anodal transcranial direct current stimulation. Exp Brain Res. 2009 Jul;196(3):459-65.

28. Madhavan S, Weber KA 2nd, Stinear JW. Non-invasive brain stimulation enhances fine motor control of the hemiparetic ankle: implications for rehabilitation. Exp Brain Res. 2011 Mar;209(1):9-17. http://dx.doi. org/10.1007/s00221-010-2511-0

29. Montenegro RA, Okano AH, Cunha FA, Fontes EB, Farinatti P. Does prefrontal cortex transcranial direct current stimulation influence the oxygen uptake at rest and postexercise? Int J Sports Med. 2013;In Press.

30. LeCheminant JD, Jacobsen DJ, Bailey BW, Mayo MS, Hill JO, Smith BK, et al. Effects of long-term aerobic exercise on EPOC. Int J Sports Med. 2008 Jan;29(1):53-8. http://dx.doi. org/10.1055/s-2007-965111.

31. Hendy AM, Kidgell DJ. Anodal tDCS applied during strength training enhances motor cortical plasticity. Med Sci Sports Exerc. 2013;45(9):1721-9. http://dx.doi.org/10.1249/ MSS.0b013e31828d2923 


\section{Autores}

\section{Ada Fernanda P. S. Lima}

Laboratório de Atividade Física e Promoção da Saúde. Instituto de Educação Física e Desportos. Universidade do Estado do Rio de Janeiro. Rio de Janeiro, RJ, Brasil.

\section{Alexandre H. Okano}

Departamento de Educação Física. Centro de Ciências da Saúde. Universidade Federal do Rio Grande do Norte. Natal, RN, Brasil.

\section{Alice R. Sampaio}

Laboratório de Atividade Física e Promoção da Saúde. Instituto de Educação Física e Desportos. Universidade do Estado do Rio de Janeiro. Rio de Janeiro, RJ, Brasil.

\section{Ana Paula M. Guttierres}

Laboratório de Atividade Física e Promoção da Saúde. Instituto de Educação Física e Desportos. Universidade do Estado do Rio de Janeiro. Rio de Janeiro, RJ, Brasil.

\section{Astrogildo V. Oliveira Júnior}

Departamento de Educação Física e Folclore. Colégio Pedro II. Rio de Janeiro, RJ, Brasil.

\section{Brenno S. Silva}

Programa de Pós-graduação em Ciências da Atividade Física. Universidade Salgado de Oliveira. Niterói, RJ, Brasil.

\section{Daniel A. Bottino}

Laboratório de Pesquisas Clínicas e Experimentais em Biologia Vascular. Centro Biomédico.

Universidade do Estado do Rio de Janeiro. Rio de Janeiro, RJ, Brasil.

\section{Dionizio Mendes Ramos Filho}

Laboratório de Bioenergética e Fisiologia Mitocondrial. Centro de Ciências da Saúde. Universidade Federal do Rio de Janeiro. Rio de Janeiro, RJ, Brasil.

\section{Elirez B. Silva}

Laboratório de Pesquisa Clínica Escola. Departamento de Fisioterapia. Universidade Gama Filho. Rio de Janeiro, RJ, Brasil.

\section{Ercole C. Rubini}

Laboratório de Fisiologia do Exercício. Universidade Estácio de Sá. Rio de Janeiro, RJ, Brasil.

\section{Fabrício V. A. Vasconcellos}

Centro de Investigação, Formação, Inovação, Intervenção e Desporto. Faculdade de Desporto. Universidade do Porto. Porto, Portugal.

\section{Felipe A. da Cunha}

Programa de Pós-graduação em Ciências Médicas. Universidade do Estado do Rio de Janeiro. Rio de Janeiro, RJ, Brasil.

\section{Fernanda Monteiro}

Laboratório de Atividade Física e Promoção da Saúde. Instituto de Educação Física e Desportos. Universidade do Estado do Rio de Janeiro. Rio de Janeiro, RJ, Brasil.

\section{Flávia Porto}

Programa de Pós-graduação em Ciências do Exercício e do Esporte. Universidade Gama Filho. Rio de Janeiro, RJ, Brasil. 


\section{Gustavo C. Lopes}

Laboratório de Atividade Física e Promoção da Saúde. Instituto de Educação Física e Desportos. Universidade do Estado do Rio de Janeiro. Rio de Janeiro, RJ, Brasil.

\section{Jonas L. Gurgel}

Programa de Pós-graduação em Ciências

Cardiovasculares. Universidade Federal Fluminense.

Niterói, RJ, Brasil.

\section{Jonathan Myers}

VA Palo Alto Health Care System. Cardiology Division. Stanford University. Palo Alto, California, United States.

\section{Karynne Grutter}

Laboratório de Anatomia Humana. Universidade Castelo Branco. Rio de Janeiro, RJ, Brasil.

\section{Lenifran Matos-Santos}

Programa de Pós-graduação em Ciências da Atividade Física. Universidade Salgado de Oliveira. Niterói, RJ, Brasil.

\section{Lorena Paes}

Programa de Pós-graduação em Fisiopatologia Clínica e Experimental. Universidade do Estado do Rio de Janeiro. Rio de Janeiro, RJ, Brasil.

\section{Luciane P. da Costa}

Laboratório de Atividade Física e Promoção da Saúde. Instituto de Educação Física e Desportos. Universidade do Estado do Rio de Janeiro. Rio de Janeiro, RJ, Brasil.

\section{Luiz G. Kraemer-Aguiar}

Departamento de Medicina Interna. Faculdade de Ciências Médicas. Universidade do Estado do Rio de Janeiro. Rio de Janeiro, RJ, Brasil.

\section{Matheus R. Hausen}

Laboratório de Atividade Física e Promoção da Saúde. Instituto de Educação Física e Desportos. Universidade do Estado do Rio de Janeiro. Rio de Janeiro, RJ, Brasil.

\section{Paulo T. V. Farinatti}

Laboratório de Atividade Física e Promoção da Saúde. Instituto de Educação Física e Desportos. Universidade do Estado do Rio de Janeiro. Rio de Janeiro, RJ, Brasil.

\section{Rafael A. Montenegro}

Programa de Pós-graduação em Fisiopatologia Clínica e Experimental. Universidade do Estado do Rio de Janeiro. Rio de Janeiro, RJ, Brasil.

\section{Raul A. Freire}

Programa de Pós-graduação em Ciências da Atividade Física. Universidade Salgado de Oliveira. Niterói, RJ, Brasil.

\section{Renato O. Massaferri}

Programa de Pós-graduação em Ciências da Atividade Física. Universidade Salgado de Oliveira. Niterói, RJ, Brasil.

\section{Ricardo B. Oliveira}

Laboratório de Atividade Física e Promoção da Saúde. Instituto de Educação Física e Desportos. Universidade do Estado do Rio de Janeiro. Rio de Janeiro, RJ, Brasil.

\section{Ricardo G. Cordeiro}

Programa de Pós-graduação em Ciências da Atividade Física. Universidade Salgado de Oliveira. Niterói, RJ, Brasil.

\section{Sérgio Machado}

Programa de Pós-graduação em Ciências da Atividade Física. Universidade Salgado de Oliveira. Niterói, RJ, Brasil.

\section{Taciana Pinto}

Laboratório de Atividade Física e Promoção da Saúde. Instituto de Educação Física e Desportos. Universidade do Estado do Rio de Janeiro. Rio de Janeiro, RJ, Brasil.

\section{Tainah P. Lima Monteiro}

Programa de Pós-graduação em Ciências Médicas. Faculdade de Ciências Médicas. Universidade do Estado do Rio de Janeiro. Rio de Janeiro, RJ, Brasil.

\section{Tânia M. P. F. Paschoalino}

Hospital Universitário Antônio Pedro. Universidade Federal Fluminense. Niterói, RJ, Brasil.

\section{Walace D. Monteiro}

Laboratório de Atividade Física e Promoção da Saúde. Instituto de Educação Física e Desportos. Universidade do Estado do Rio de Janeiro. Rio de Janeiro, RJ, Brasil. 